\title{
Norm minima in certain Siegel leaves
}

\author{
LI CAI
}

In this paper we shall illustrate that each polytopal moment-angle complex can be understood as the intersection of the minima of corresponding Siegel leaves and the unit sphere, with respect to the maximum norm. Consequently, an alternative proof of a rigidity theorem of Bosio and Meersseman is obtained; as piecewise linear manifolds, polytopal real moment-angle complexes can be smoothed in a natural way.

57R30; 57R70, 05E45

\section{Introduction}

An admissible configuration of $m$ complex vectors in $\mathbb{C}^{d / 2}(m>d$ with $d$ even) satisfying so called Siegel and weak hyperbolicity conditions (see Meersseman [15, page 82], and Section 2 for a real analogue), gives rise to a free action on $\mathbb{C}^{m}$ via exponential functions. There are two types of leaves in the holomorphic foliation given by this action: a leaf is of Siegel type if the origin is not in its closure; otherwise it is said to be of Poincaré type.

These objects originated in the work of C Camacho, N Kuiper and J Palls [6] on the complex analogue of a dynamical system for which the real version appeared in an earlier work of Poincaré, and were later developed and generalized by S López de Medrano and A Verjovsky [14] and L Meersseman [15]. From their works, the projectivization of the minima of all Siegel leaves, with respect to the Euclidean norm, can be endowed with the structure of a compact, complex $(m-d / 2-1)$-manifold $C^{\infty}$-embedded in $\mathbb{C} P^{m-1}$, which is not symplectic except in the trivial case. This class of complex manifolds is now named LVM manifolds.

On the other hand, by a direct calculation, the space of minima of all Siegel leaves can be described by $d$ real quadrics arising from the given configuration in $\mathbb{R}^{d}$, whose intersection with the unit Euclidean sphere in $\mathbb{C}^{m}$ is transverse, hence it is a smooth manifold of real dimension $2 m-d-1$. F Bosio and L Meersseman [3] observed that this method also works for odd $d$, and call these manifolds embedded in spheres links.

This special class of links is a model for polytopal moment-angle manifolds. In general their topology is known to be complicated (see [3] and Gitler and López de 
Medrano [10]), for instance, arbitrary torsion can appear in the cohomology, as well as nonvanishing triple Massey products (see Baskakov [2] and Denham and Suciu [9]); in the case $d=2$, the classification work [13] by S López de Medrano shows that they are diffeomorphic to a triple product of spheres or to the connected sum of sphere products. An important way to understand them is that they inherit the natural $\left(S^{1}\right)^{m}$-action on $\mathbb{C}^{m}$, with each quotient space homeomorphic (as manifolds with corners) to a simple convex polytope. Via the basic construction originating from reflection group theory and then generalized by M W Davis and T Januszkiewicz in their influential work [8], each link discussed above is homeomorphic to a moment-angle complex (named in Buchstaber and Panov [5]), ie a polyhedral product with pairs $\left(D^{2}, S^{1}\right)$ corresponding to the boundary complex of a simplicial polytope.

The polyhedral product model was studied in detail and generalized by V Buchstaber and T Panov in [5]. Later a more categorical treatment by A Bahri, M Bendersky, F R Cohen and S Gitler [1] provided a penetrating viewpoint from homotopy theory.

These spaces have spawned a large body of work; see most notably that by Davis and Januszkiewicz [8] on quasitoric varieties, Buchstaber and Panov [5] on moment-angle complexes, Goresky and MacPherson [11] on complements of complex arrangements, S López de Medrano [13] on the topology of these varieties, as well as many others. The interconnections between these subjects is developed in the beautiful book [4] by Buchstaber and Panov.

The objective of this paper is to show that, for an admissible configuration of $m$ real vectors in $\mathbb{R}^{d}$ whose centroid is located at the origin, the corresponding foliation provides a direct relation between the model of links and the model of polyhedral products: there are continuous paths in the space of the union of all Siegel leaves (which is the complement of a coordinate subspace arrangement in $\mathbb{C}^{m}$ ) such that each point of the link is connected by a path to a unique point in the respective moment-angle complex, yielding a homeomorphism between them. Every path is parameterized by real numbers $p \in[1, \infty)$, with each $p$ associated to the intersection of the $L^{p}$-norm minima in the Siegel leaves and the $L^{p}$-norm unit sphere in $\mathbb{C}^{m}$, which is a topological manifold homeomorphic to the link. In this way, we can understand each polytopal moment-angle complex as the intersection of the unit sphere and the minima of all Siegel leaves, with respect to the $L^{\infty}$-norm.

This paper develops a more analytic approach to these spaces in the spirit of the work [3] by Bosio and Meersseman.

I would like to thank my PhD supervisor, Professor Osamu Saeki, for many discussions. 


\section{Notation and main results}

Let $A=\left(A_{1}, A_{2}, \ldots, A_{m}\right)$ be an $m$-tuple of vectors in $\mathbb{R}^{d}$, with $m>d \geq 0\left(A_{i} \equiv 0\right.$ when $d=0)$; occasionally we treat such a tuple as a $(d \times m)$-matrix. Denote by $[m]$ the set $\{1,2, \ldots, m\}$, and for $I \subset[m]$, let $A(I)$ be the subtuple $\left(A_{i}\right)_{i \in I}$ and $\operatorname{conv} A$ (resp. conv $A(I))$ the convex hull of vectors from $A$ (resp. from $A(I)$ ).

We say $A$ is admissible if it satisfies the following two conditions (cf [3, Lemma 0.3]):

$*_{1} \quad$ (Siegel condition) $\mathbf{0} \in \operatorname{conv} A$.

$*_{2}$ (Weak hyperbolicity condition) If $\mathbf{0} \in \operatorname{conv} A(I)$, then we have $\operatorname{card}(I)>d$ (where card refers to the cardinality).

Up to Section 5, we always assume that $A$ is admissible.

Let $\mathbb{R}_{>0}$ be the set of positive real numbers, in which $p \geq 1$ is a real number. For each $z=\left(z_{i}\right)_{i=1}^{m} \in \mathbb{C}^{m}$, denote by $\|z\|_{p}$ its $L^{p}$-norm, namely $\|z\|_{p}=\left(\sum_{i=1}^{m}\left|z_{i}\right|^{p}\right)^{1 / p}$, where $\left|z_{i}\right|=\sqrt{z_{i} \bar{z}_{i}}$.

With respect to an $m$-tuple $A$, there is a smooth foliation $\mathcal{F}$ of $\mathbb{C}^{m}$ given by the orbits of the action

$$
\begin{aligned}
F: \mathbb{C}^{m} \times \mathbb{R}^{d} & \rightarrow \mathbb{C}^{m}, \\
(z, T) & \mapsto\left(z_{i} e^{\left\langle A_{i}, T\right\rangle}\right)_{i=1}^{m} .
\end{aligned}
$$

For each $z \in \mathbb{C}^{m}$, let $L_{z}$ be the leaf passing through $z$. We call $L_{z}$ a Siegel leaf if $\mathbf{0}$ is not in its closure, otherwise we say the leaf $L_{z}$ is of Poincaré type. It follows that the union of all Siegel leaves can be described by the set (see [6;3] and Meersseman and Verjovsky [16])

$$
\mathcal{S}_{A}=\left\{z \in \mathbb{C}^{m} \mid \mathbf{0} \in \operatorname{conv} A\left(I_{z}\right)\right\},
$$

where $I_{z}$ is the set of nonzero entries for $z=\left(z_{i}\right)_{i=1}^{m}$, ie $I_{z}=\left\{i \in[m]|| z_{i} \mid \neq 0\right\}$. With an argument involving foliations, complex analysis and the convexity, the following fact is a combination of the works mentioned above, which is our starting point:

Theorem 1 (cf [3, Lemma 0.8, pages 61-62]) For each $z \in \mathcal{S}_{A}$, there is a unique point $f_{2}(z)$ in the leaf $L_{z}$ such that its $L^{2}$-norm $\left\|f_{2}(z)\right\|_{2}$ is minimal and positive. The foliation $\mathcal{F}$ is trivial when restricted to $\mathcal{S}_{A}$, and

$$
\begin{aligned}
\Phi_{A}(2): X_{A}(2) \times \mathbb{R}^{d} \times \mathbb{R}_{>0} & \rightarrow \mathcal{S}_{A}, \\
(z, T, r) & \mapsto r\left(z_{i} e^{\left\langle A_{i}, T\right\rangle}\right)_{i=1}^{m},
\end{aligned}
$$


is a global diffeomorphism, where $X_{A}(2)$ is given by the transverse intersection

$$
\left\{\begin{array}{l}
\sum_{i=1}^{m} A_{i}\left|z_{i}\right|^{2}=\mathbf{0}, \\
\|z\|_{2}=1,
\end{array}\right.
$$

and is thus a smooth manifold.

It follows that there is a smooth function

$$
T_{2}: \mathcal{S}_{A} \rightarrow \mathbb{R}^{d} \quad \text { such that } f_{2}(z)=F\left(z, T_{2}(z)\right),
$$

and after differentiating $F(z, T)$ with respect to $T \in \mathbb{R}^{d}$, one easily checks that the critical point corresponding to the minimum satisfies

$$
\sum_{i=1}^{m} A_{i}\left|z_{i}\right|^{2} e^{2\left\langle A_{i}, T\right\rangle}=\mathbf{0},
$$

in which $T_{2}(z)$ is the unique solution. Moreover, $f_{2} /\left\|f_{2}\right\|_{2}: \mathcal{S}_{A} \rightarrow X_{A}(2)$ is a smooth retraction.

Following their approach, we consider the space of $L^{p}$-norm minima of those Siegel leaves. Our first main theorem is the following, whose proof is based on some real analysis and will be given in Section 3 .

Theorem 2 Let $X_{A}(p)$ be the intersection

$$
\left\{\begin{array}{l}
\sum_{i=1}^{m} A_{i}\left|z_{i}\right|^{p}=\mathbf{0}, \\
\|z\|_{p}=1 .
\end{array}\right.
$$

There is a unique point $f_{p}(z)$ in the leaf $L_{z}$ for each element $z \in \mathcal{S}_{A}$, whose $L^{p}$ norm $\left\|f_{p}(z)\right\|_{p}$ is minimal and positive, and the restriction of the smooth function $f_{2} /\left\|f_{2}\right\|_{2}: S_{A} \rightarrow X_{A}(2)$ to $X_{A}(p)$ induces a homeomorphism onto $X_{A}(2)$ for all $p \geq 1$. Moreover,

$$
\begin{aligned}
\Phi_{A}(p): X_{A}(p) \times \mathbb{R}^{d} \times \mathbb{R}_{>0} & \rightarrow \mathcal{S}_{A}, \\
(z, T, r) & \mapsto r\left(z_{i} e^{\left\langle A_{i}, T\right\rangle}\right)_{i=1}^{m},
\end{aligned}
$$

is a homeomorphism.

Similar to (4), for each $p$ we can define a continuous function $T_{p}: \mathcal{S}_{A} \rightarrow \mathbb{R}^{d}$ such that $f_{p} /\left\|f_{p}\right\|_{p}: \mathcal{S}_{A} \rightarrow X_{A}(p)$ is a retraction, where $f_{p}(z)=F\left(z, T_{p}(z)\right)$ is the function of $L^{p}$-norm minima in the leaf $L_{z}$. 
It is interesting to imagine what will happen when $p$ tends to infinity, and we will discuss this in Section 4. First note that the set

$$
K_{A}=\{\sigma \subset[m] \mid \mathbf{0} \in \operatorname{conv} A([m] \backslash \sigma)\}
$$

is an abstract simplicial complex (see [3, Lemma 0.12]), ie all subsets of $\sigma$ will be in $K_{A}$ if $\sigma$ is. It turns out that with each $z \in \mathcal{S}_{A}$ fixed, $T_{p}(z)$ and $f_{p}(z) /\left\|f_{p}(z)\right\|_{p}$ are continuous in $p \in[1, \infty)$ (see Proposition 4.2); when $p$ goes to infinity, $f_{p}(z) /\left\|f_{p}(z)\right\|_{p}$ approaches the moment-angle complex $\left(D^{2}, S^{1}\right) K_{A}$ (see Section 4.1 and Proposition 4.4 for details), which is a subset of the intersection of $\mathcal{S}_{A}$ with the $L^{\infty}$-norm unit sphere in $\mathbb{C}^{m}\left(\|z\|_{\infty}=\max \left\{\left|z_{i}\right|\right\}_{i=1}^{m}\right)$.

We say that the tuple $A$ is centered at the origin if the centroid of all vectors in $A$ are located at the origin:

$$
\sum_{i=1}^{m} A_{i}=\mathbf{0} .
$$

Under this additional assumption, $K_{A}$ is isomorphic to the boundary of a convex polytope arising from the Gale transform of $A$ (see Proposition 5.3); based on a result of Panov and Ustinovsky [18], in Section 5 we will show that $f_{p}(z) /\left\|f_{p}(z)\right\|_{p}$ converges to a unique point in $\left(D^{2}, S^{1}\right)^{K_{A}}$ as $p$ tends to infinity. With a similar treatment as the one for Theorem 2, the following theorem holds:

Theorem 3 Assume that $A$ is an admissible tuple centered at the origin. Then the restriction $f_{2} /\left.\left\|f_{2}\right\|_{2}\right|_{\left(D^{2}, S^{1}\right)^{K_{A}}}:\left(D^{2}, S^{1}\right)^{K_{A}} \rightarrow X_{A}(2)$ is a homeomorphism. Moreover,

$$
\begin{aligned}
\Phi_{A}(\infty):\left(D^{2}, S^{1}\right)^{K_{A}} \times \mathbb{R}^{d} \times \mathbb{R}_{>0} & \rightarrow \mathcal{S}_{A}, \\
(z, T, r) & \mapsto r\left(z_{i} e^{\left\langle A_{i}, T\right\rangle}\right)_{i=1}^{m},
\end{aligned}
$$

is a homeomorphism.

Therefore, we can understand such a moment-angle complex $\left(D^{2}, S^{1}\right)^{K_{A}}$ as " $X_{A}(\infty)$ ", namely the intersection of the $L^{\infty}$-norm minima in the Siegel leaves with the $L^{\infty}$ norm unit sphere in $\mathbb{C}^{m}$ (the reader is encouraged to imagine the deformation from $X_{A}(1)$ to $X_{A}(\infty)$ in the case $\left.d=0\right)$.

As an application, in Section 6 we give an alternative proof for a rigidity theorem of Bosio and Meersseman [3, Theorem 4.1]: if two admissible $m$-tuples $A$ and $A^{\prime}$ are both centered at the origin such that $K_{A}$ and $K_{A^{\prime}}$ are isomorphic simplicially, then there is a diffeomorphism between associated links $X_{A}(2)$ and $X_{A^{\prime}}$ (2) (see Proposition 6.1 for more details). 
From its definition (1), notice that each leaf $L_{z}$ is contained in $\mathcal{S}_{A} \cap \mathbb{R}^{m}$ if and only if $z \in \mathcal{S}_{A} \cap \mathbb{R}^{m}$. Hence the theorems and properties above are also true when restricted to the subspace $\mathbb{R}^{m}$ in $\mathbb{C}^{m}$.

At last in Section 6, we shall illustrate that the restriction of $f_{2} /\left\|f_{2}\right\|_{2}$ to the real moment-angle complex $\left(D^{1}, S^{0}\right)^{K_{A}}=\left(D^{2}, S^{1}\right)^{K_{A}} \cap \mathbb{R}^{m}$ is a piecewise differentiable homeomorphism onto $X_{A}(2) \cap \mathbb{R}^{m}$, provided that $A$ is admissible and centered at the origin (see Definition 6.2, Lemma 6.3 and Proposition 6.4 for more details). In this way these real moment-angle complexes can be smoothed as piecewise linear manifolds.

\section{Proof of Theorem 2}

We start with a well-known lemma due to Meersseman and Verjovsky, whose proof is omitted here:

Lemma 3.1 $\left[16\right.$, Lemma 1.1; 3, Lemma 0.3] For an admissible tuple $A=\left(A_{i}\right)_{i=1}^{m}$, let $\widetilde{A}=\left(\widetilde{A}_{i}\right)_{i=1}^{m}$ be the augmentation with $\widetilde{A}_{i}=\left(A_{i}^{\mathrm{T}}, 1\right)^{\mathrm{T}} \in \mathbb{R}^{d+1}, i=1,2, \ldots, m$. Then for any $I \subset[m]$ such that $\mathbf{0} \in \operatorname{conv} A(I)$, the rank of the subtuple $\tilde{A}(I)$ is $d+1$.

Proposition 3.2 For each $z \in \mathcal{S}_{A}$ given, there is a unique point $f_{p}(z)$ in the leaf $L_{z}$ such that $\left\|f_{p}(z)\right\|_{p}$ is minimal and positive.

Proof Uniqueness (cf $[6 ; 15 ; 16])$ Assume $F_{z}$ has two local minima, ie $T_{1}$ and $T_{2}$ in $\mathbb{R}^{d}$ that are both critical points of $\left(\|F(z, T)\|_{p}\right)^{p}=\sum_{i=1}^{m}\left|z_{i}\right|^{p} e^{p\left\langle A_{i}, T\right\rangle}$, which means

$$
\sum_{i=1}^{m} A_{i}\left|z_{i}\right|^{p} e^{p\left\langle A_{i}, T_{j}\right\rangle}=\mathbf{0}, \quad j=1,2 .
$$

We define a function $h:[0,1] \rightarrow \mathbb{R}$ such that $h(t)=\left(\left\|F\left(z,(1-t) T_{1}+t T_{2}\right)\right\|_{p}\right)^{p}$; clearly

$$
\frac{\mathrm{d} h}{\mathrm{~d} t}=p \sum_{i=1}^{m}\left\langle A_{i}, T_{2}-T_{1}\right\rangle\left|z_{i}\right|^{p} e^{p\left\langle A_{i},(1-t) T_{1}+t T_{2}\right\rangle} .
$$

From Lemma 3.1, the subtuple $A\left(I_{z}\right)$ has rank $d\left(I_{z} \subset[m]\right.$ consists of entries $i$ such that $z_{i} \neq 0$ ), which is independent of $z \in \mathcal{S}_{A}$, thus there exists $i \in I_{z}$ such that $\left\langle A_{i}, T_{2}-T_{1}\right\rangle$ does not vanish; it follows that the second derivative of $h$ is strictly positive, hence its first derivative (9) is strictly increasing, which is a contradiction.

Existence First from the Cauchy-Schwarz inequality

$$
\|F(z, T)\|_{2} \leq\|F(z, T)\|_{1} \leq \sqrt{m}\|F(z, T)\|_{2},
$$


together with Theorem 1 and Lemma 3.3 below, we conclude that $\|F(z, T)\|_{1}$ bounds away from zero, and stays large whenever $\|T\|_{2}$ is large. Thus the minimum of $\|F(z, T)\|_{1}$ is positive, and it appears only when $T$ is in the interior of a ball of finite radius. So the case $p=1$ is clear. For general cases when $p \neq 1,2$, Hölder's inequality implies

$$
\|F(z, T)\|_{p} \leq\|F(z, T)\|_{1} \leq \sqrt[q]{m}\|F(z, T)\|_{p}
$$

here $q>1$ such that $1 / p+1 / q=1$. We can repeat the previous argument and then the proof is completed.

Lemma 3.3 With $z \in \mathcal{S}_{A}$ given, for any $N>0$, there exists $R>0$ such that $\|F(z, T)\|_{2}>N$ whenever $\|T\|_{2}>R$.

Proof Let $T_{2}(z)$ be the point in $\mathbb{R}^{d}$ such that $\left\|F\left(z, T_{2}(z)\right)\right\|_{2}$ is minimal (see (4) for details). Denote by $u\left(t ; T_{1}, T_{2}\right)$ the derivative of $\left(\left\|F\left(z,(1-t) T_{1}+t T_{2}\right)\right\|_{2}\right)^{2}$ with respect to $t \in[0,1]$, for $T_{1}, T_{2} \in \mathbb{R}^{d}$, and let $B\left(r, T_{2}(z)\right)$ be the ball with radius $r$ centered at $T_{2}(z)$. Since $T_{2}(z)$ is the unique minimum, for all $y \in \partial B\left(1, T_{2}(z)\right)$ on the boundary, there is a positive $\varepsilon$ such that

$$
\left(\|F(z, y)\|_{2}\right)^{2}-\left(\left\|F\left(z, T_{2}(z)\right)\right\|_{2}\right)^{2}=\int_{0}^{1} u\left(t ; T_{2}(z), y\right) d t>\varepsilon ;
$$

therefore we can choose $t(y) \in(0,1)$ such that

$$
u\left(t(y) ; T_{2}(z), y\right)>\varepsilon,
$$

by the mean value theorem. For $r>1$, assume $y_{r} \in \partial B\left(r, T_{2}(z)\right)$ with $y \in \partial B\left(1, T_{2}(z)\right)$ on the ray from $T_{2}(z)$ to $y_{r}$; by the monotonicity of $u\left(t ; T_{2}, y_{r}\right)$ (see the uniqueness part in the proof of Proposition 3.2), we have

$$
u\left(t ; y, y_{r}\right)>u\left(t(y) ; T_{2}, y\right),
$$

thus

$$
\begin{aligned}
& \left(\left\|F\left(z, y_{r}\right)\right\|_{2}\right)^{2}-\left(\left\|F\left(z, T_{2}(z)\right)\right\|_{2}\right)^{2} \\
& \quad=\int_{0}^{1} u\left(t ; T_{2}, y\right) d t+\int_{0}^{1} u\left(t ; y, y_{r}\right) d t>\varepsilon+(r-1) \varepsilon,
\end{aligned}
$$

from which the conclusion follows.

The function of minima $f_{p}: \mathcal{S}_{A} \rightarrow \mathcal{S}_{A}$ is well-defined by Proposition 3.2; but except for the case $p=2$, it remains to prove its continuity. In what follows we shall illustrate this by showing the continuity of the restriction $f_{p} /\left.\left\|f_{p}\right\|_{p}\right|_{X_{A}(2)}$ first, and then it will follow from the global diffeomorphism $\Phi_{A}(2)$ defined in Theorem 1. 
Proposition 3.4 The restriction $f_{2} /\left.\left\|f_{2}\right\|_{2}\right|_{X_{A}(p)}$ of the smooth function

$$
f_{2} /\left\|f_{2}\right\|_{2}: S_{A} \rightarrow X_{A}(2)
$$

induces a homeomorphism onto $X_{A}(2)$, whose inverse is

$$
f_{p} /\left.\left\|f_{p}\right\|_{p}\right|_{X_{A}(2)}: X_{A}(2) \rightarrow X_{A}(p)
$$

Proof Consider the function

$$
\begin{aligned}
\Phi_{A}: \mathcal{S}_{A} \times \mathbb{R}^{d} \times \mathbb{R}_{>0} & \rightarrow \mathcal{S}_{A}, \\
(z, T, r) & \mapsto r\left(z_{i} e^{\left\langle A_{i}, T\right\rangle}\right)_{i=1}^{m},
\end{aligned}
$$

from Theorem 1 and Proposition 3.2. Given $z \in \mathcal{S}_{A}$, its image under $\Phi_{A}$ intersects both $X_{A}(p)$ and $X_{A}(2)$ exactly once, respectively, hence $f_{2} /\left.\left\|f_{2}\right\|_{2}\right|_{X_{A}}(p)$ is a bijection. Moreover, it is easy to see that $X_{A}(p)$ is compact and $X_{A}(2)$ is Hausdorff; since a closed subspace of a compact space is compact, and a compact subspace of a Hausdorff space is closed, it follows that $f_{2} /\left.\left\|f_{2}\right\|_{2}\right|_{X_{A}}(p)$ is closed and hence a homeomorphism by the bijectiveness. As a conclusion, its inverse $f_{p} /\left.\left\|f_{p}\right\|_{p}\right|_{X_{A}(2)}$ is continuous.

Theorem 3.5 The continuous function

$$
\begin{aligned}
\Phi_{A}(p): X_{A}(p) \times \mathbb{R}^{d} \times \mathbb{R}_{>0} & \rightarrow \mathcal{S}_{A}, \\
(z, T, r) & \mapsto r\left(z_{i} e^{\left\langle A_{i}, T\right\rangle}\right)_{i=1}^{m},
\end{aligned}
$$

is a homeomorphism for all $p \geq 1$.

Proof It suffices to find a continuous inverse for $\Phi_{A}(p)$. Suppose $f_{p}(z) /\left\|f_{p}(z)\right\|_{p}=$ $\left(x_{i}(z)\right)_{i=1}^{m}$. For $(z, T, r) \in X_{A}(2) \times \mathbb{R}^{d} \times \mathbb{R}_{>0}$, we can rewrite

$$
\begin{aligned}
z & =\rho^{-1}(z) F\left(f_{p}(z) /\left\|f_{p}(z)\right\|_{p}, T_{2}\left(f_{p}(z) /\left\|f_{p}(z)\right\|_{p}\right)\right) \\
& =\rho^{-1}(z)\left(x_{i}(z) e^{\left\langle A_{i}, T_{2}\left(f_{p}(z) /\left\|f_{p}(z)\right\|_{p}\right)\right\rangle}\right)_{i=1}^{m},
\end{aligned}
$$

where $\rho(z)=\left\|\left(x_{i}(z) e^{\left\langle A_{i}, T_{2}\left(f_{p}(z) /\left\|f_{p}(z)\right\|_{p}\right)\right\rangle}\right)_{i=1}^{m}\right\|_{2}$. The continuity of $\rho^{-1}(z), x_{i}(z)$ and $e^{\left\langle A_{i}, T_{2}\left(f_{p}(z) /\left\|f_{p}(z)\right\|_{p}\right)\right\rangle}$ follows from Proposition 3.4 (by Theorem 1, $T_{2}$ is smooth).

Observe that

$$
\begin{aligned}
\Phi_{A}(2)(z, T, r) & =r\left(z_{i} e^{\left\langle A_{i}, T\right\rangle}\right)_{i=1}^{m}=r \rho^{-1}(z)\left(x_{i}(z) e^{\left\langle A_{i}, T+T_{2}\left(f_{p}(z) /\left\|f_{p}(z)\right\|_{p}\right)\right\rangle}\right)_{i=1}^{m} \\
& =\Phi_{A}(p)\left(f_{p}(z) /\left\|f_{p}(z)\right\|_{p}, T+T_{2}\left(f_{p}(z) /\left\|f_{p}(z)\right\|_{p}\right), r \rho^{-1}(z)\right),
\end{aligned}
$$


hence we have a coordinate transition function

$$
\begin{aligned}
\varphi: X_{A}(2) \times \mathbb{R}^{d} \times \mathbb{R}_{>0} & \rightarrow X_{A}(p) \times \mathbb{R}^{d} \times \mathbb{R}_{>0}, \\
(z, T, r) & \mapsto\left(f_{p}(z) /\left\|f_{p}(z)\right\|_{p}, T+T_{2}\left(f_{p}(z) /\left\|f_{p}(z)\right\|_{p}\right), r \rho^{-1}(z)\right) .
\end{aligned}
$$

It is straightforward to check the continuity of $\varphi$, thus $\varphi \circ\left(\Phi_{A}(2)\right)^{-1}$ is the inverse of $\Phi_{A}(p)$.

Corollary 3.6 The function

$$
T_{p}: \mathcal{S}_{A} \rightarrow \mathbb{R}^{d} \text { such that } f_{p}(z)=F\left(z, T_{p}(z)\right)
$$

is well-defined and continuous. That is to say, for each $z \in \mathcal{S}_{A}, T_{p}(z)$ is the unique solution of the equation

$$
\sum_{i=1}^{m} A_{i}|z|_{i}^{p} e^{p\left\langle A_{i}, T\right\rangle}=\mathbf{0},
$$

which depends continuously on $z$.

\section{When $p$ tends to infinity}

In this section we treat $T_{p}(z)$ and $f_{p}(z)$ (defined in Corollary 3.6 and Proposition 3.2 respectively) as functions of $p \in[1, \infty)$, with $z \in \mathcal{S}_{A}$ fixed.

Lemma 4.1 There exists a bound $N(z)$ such that $\left\|T_{p}(z)\right\|_{2}<N(z)$ for all $p \in[1, \infty)$.

Proof By definition, $\left\|F\left(z, T_{p}(z)\right)\right\|_{p}$ is the unique minimum in the leaf $L_{z}$. Suppose that on the contrary, there exists a sequence $\left\{p_{k}\right\}_{k=1}^{\infty}$ tending to infinity such that $\left\|T_{p_{k}}(z)\right\|_{2}>k$ for each $k$. First by Lemma 3.3 and the Cauchy-Schwarz inequality (10), $\|F(z, T)\|_{1}$ becomes arbitrarily large whenever $\|T\|_{2}$ is large enough, thus there exists $N>0$ such that for all $k>N, m\left\|F\left(z, T_{1}(z)\right)\right\|_{1}<\left\|F\left(z, T_{p_{k}}(z)\right)\right\|_{1}$. Then by Hölder's inequality (11), we have

$$
\begin{aligned}
\sqrt[q_{k}]{m}\left\|F\left(z, T_{1}(z)\right)\right\|_{p_{k}} \leq \sqrt[q_{k}]{m}\left\|F\left(z, T_{1}(z)\right)\right\|_{1} & <\left\|F\left(z, T_{p_{k}}(z)\right)\right\|_{1} \\
& \leq \sqrt[q_{k}]{m}\left\|F\left(z, T_{p_{k}}(z)\right)\right\|_{p_{k}},
\end{aligned}
$$

where $1 / p_{k}+1 / q_{k}=1$. It follows that $\left\|F\left(z, T_{p_{k}}(z)\right)\right\|_{p_{k}}$ is strictly greater than $\left\|F\left(z, T_{1}(z)\right)\right\|_{p_{k}}$, yielding a contradiction.

Proposition 4.2 The function $T_{p}(z)$ is continuous for all $p \in[1, \infty)$. 
Proof Suppose again on the contrary there is a sequence $\left\{p_{k}\right\}_{k=1}^{\infty}$ with $\lim _{k} p_{k}=p_{0}$, but $\left\|T_{p_{k}}(z)-T_{p_{0}}(z)\right\|_{2} \geq \delta$, for some $\delta>0$. Without loss of generality we may assume that $\lim _{k} T_{p_{k}}=T_{0} \neq T_{p_{0}}(z)$, or we can choose a subsequence satisfying the property, by the lemma above. Consider the smooth function

$$
\begin{aligned}
\mu:[1, \infty) \times \mathbb{R}^{d} & \rightarrow \mathbb{R}^{d}, \\
(p, T) & \mapsto \sum_{i=1}^{m} A_{i}\left|z_{i}\right|^{p} e^{p\left\langle A_{i}, T\right\rangle} ;
\end{aligned}
$$

we have $\mathbf{0}=\lim _{k} \mu\left(p_{k}, T_{k}(z)\right)=\mu\left(p_{0}, T_{0}\right)$ by continuity, contradicting the uniqueness (see Corollary 3.6).

Corollary 4.3 As a function of $p \in[1, \infty), f_{p}(z) /\left\|f_{p}(z)\right\|_{p}$ is continuous with its image in the $L^{p}$-link $X_{A}(p)$ (defined by (6)), and we have

$$
\lim _{p \rightarrow \infty}\left\|f_{p}(z) /\right\| f_{p}(z)\left\|_{p}\right\|_{\infty}=1 .
$$

Proof Denote $f_{p}(z) /\left\|f_{p}(z)\right\|_{p}$ by $y(p)=\left(y_{i}(p)\right)_{i=1}^{m}$. Observe that

$$
1=\|y(p)\|_{p}=\|y(p)\|_{\infty}\left(\sum_{i=1}^{m}\left|\frac{y_{i}(p)}{\|y(p)\|_{\infty}}\right|^{p}\right)^{1 / p},
$$

where the last term in the bracket does not exceed $m$, thus (13) holds as desired.

\subsection{Moment-angle complexes}

Let $K_{A}$ be the simplicial complex defined by (7). The associated moment-angle complex $\left(D^{2}, S^{1}\right)^{K_{A}}$ is defined as the polyhedral product

$$
\begin{aligned}
& \left(D^{2}, S^{1}\right)^{K_{A}}=\bigcup_{\sigma \in K_{A}} D(\sigma), \quad D(\sigma)=\prod_{i=1}^{m} Y_{i}, \\
& Y_{i}= \begin{cases}D^{2}=\{|z| \leq 1 \mid z \in \mathbb{C}\} & \text { if } i \in \sigma, \\
S^{1}=\{|z|=1 \mid z \in \mathbb{C}\} & \text { otherwise. }\end{cases}
\end{aligned}
$$

The proposition below implies that $f_{p}(z) /\left\|f_{p}(z)\right\|_{p} \in X_{A}(p)$ approaches $\left(D^{2}, S^{1}\right)^{K_{A}}$ as $p$ tends to infinity.

Proposition 4.4 Let $S_{\infty}$ be the unit sphere of $\mathbb{C}^{m}$ with respect to the $L^{\infty}$-norm, and let $z \in \mathcal{S}_{A}$ be a given point. Then for every point $z^{\prime}=\left(z_{i}^{\prime}\right)_{i=1}^{m} \in S_{\infty} \cap \mathcal{S}_{A} \backslash\left(D^{2}, S^{1}\right)^{K_{A}}$, $f_{p}(z) /\left\|f_{p}(z)\right\|_{p}$ will go outside of the set

$$
C\left(z^{\prime}\right)=\left\{\left(z_{i}\right)_{i=1}^{m} \in \mathbb{C}^{m}|| z_{i}|\leq| z_{i}^{\prime} \mid \text { for all } i=1,2, \ldots, m\right\},
$$

whenever $p$ is sufficiently large. 
Proof Denote by $B \subset \mathbb{R}^{d}$ the union of all convex hulls of the form $\operatorname{conv} A([m] \backslash \tau)$ with $\tau \subset[m]$ not contained in $K_{A}$ (in other words, $\mathbf{0} \notin \operatorname{conv} A([m] \backslash \tau)$ ). It is clear that $B$ is empty when and only when $K_{A}$ bounds the $(m-1)-$ simplex (ie $K_{A}=$ $2^{[m]} \backslash[m]$, this happens only when $d=0$, by the admissibility of $A$ ), which means $S_{\infty}=\left(D^{2}, S^{1}\right)^{K_{A}}$ and we have nothing to prove; otherwise $B$ is compact thus there is an open neighborhood $U_{B}$ such that $\mathbf{0} \notin U_{B}$.

Suppose the contrary, namely there is a sequence $\left\{p_{k}\right\}_{k=1}^{\infty}$ tending to infinity such that $x_{k}=\left(x_{k i}\right)_{i=1}^{m}=f_{p_{k}}(z) /\left\|f_{p_{k}}(z)\right\|_{p} \in C\left(z^{\prime}\right)$. Since $C\left(z^{\prime}\right)$ is compact, we may assume that $\left\{x_{k}\right\}_{k=1}^{\infty}$ converges to a point $x_{0}=\left(x_{0 i}\right)_{i=1}^{m} \in C\left(z^{\prime}\right)$, without loss of generality.

We claim that the vector

$$
\sum_{i=1}^{m} A_{i}\left|x_{k i}\right|^{p_{k}}
$$

lies in $U_{B}$ whenever $k$ is large enough. Notice that this will be a contradiction since $x_{k} \in X_{A}(p)$, whose definition implies that the vector above should always be zero.

To see this, first note that because $x_{0} \notin\left(D^{2}, S^{1}\right)^{K_{A}}$, there exists $\tau \notin K_{A}$, such that $\left|x_{0 i}\right|<\delta<1$ for all $i \in \tau$. This means for those $i \in \tau$, there exists an $N>0$ such that $\left|x_{k i}\right|<\delta<1$ holds when $k>N$; thus for any given $\varepsilon>0$, we can find $N_{\varepsilon}>N$ such that $\left|x_{k i}\right|^{p_{k}}<\varepsilon$ for all $k>N_{\varepsilon}$. It is not difficult to see that, if $\varepsilon$ is small enough, vector (14) shall lie in $U_{B}$, as claimed.

\section{The convergence}

In this section we shall prove that the function $f_{p}(z) /\left\|f_{p}(z)\right\|: \mathcal{S}_{A} \rightarrow X_{A}(p)$ indeed converges to a point in $\left(D^{2}, S^{1}\right)^{K_{A}}$, as one may expect from Proposition 4.4, with an additional assumption that $A$ is centered at the origin (see (8)). The main technique we use here is combinatorial, in which Gale transforms play an essential role. ${ }^{1}$

Suppose $V=\left(V_{1}, V_{2}, \ldots, V_{m}\right)$ is a tuple of vectors in $\mathbb{R}^{m-d-1}$ such that the affine dimension of $V$ is $m-d-1$, ie the matrix with columns $\left(V_{i}^{\mathrm{T}}, 1\right)^{\mathrm{T}}(i=1,2, \ldots, m)$ has rank $m-d$.

Denote by $A_{V}=\left(A_{1}, A_{2}, \ldots, A_{m}\right)$ the Gale transform of $V$ (see Grünbaum [12, Chapter 5.4, pages 85-86]), which is the transpose of a basis of solutions of the following

\footnotetext{
${ }^{1}$ I would like to thank the referee for pointing out that analogues of Lemma 5.2 and Proposition 5.3 are already proven in $[16 ; 3]$, where Gale transforms have been intensely used. The approach here is motivated by those in these works.
} 
linear system:

$$
\left\{\begin{array}{l}
\sum_{i=1}^{m} V_{i} x_{i}=0, \\
\sum_{i=1}^{m} x_{i}=0 .
\end{array}\right.
$$

It is clear that each $A_{i}$ is a vector in $\mathbb{R}^{d}$ and different choices of $A_{V}$ are linearly equivalent.

Recall that for any $J \subset[m]$, the subtuple $V(J)=\left(V_{i}\right)_{i \in J}$ is a face of $V$ if the intersection of conv $V([m] \backslash J)$ with the affine space spanned by vectors in $V(J)$ is empty (see [12, Chapter 5.4, page 88]). For instance, if $V$ consists of the vertices of a convex polytope $P$, then $V(J)$ is a face of $V$ when and only when conv $V(J)$ is a face of $P$. Now we need two facts about Gale transforms:

Proposition 5.1 [12, Chapter 5.4, page 88] Let $V=\left(V_{i}\right)_{i=1}^{m}$ be a tuple of vectors in $\mathbb{R}^{m-d-1}$, whose affine dimension is $m-d-1$, and let $A_{V}=\left(A_{i}\right)_{i=1}^{m}$ be its Gale transform.

Then for any $I \subset[m]$, conv $V([m] \backslash I)$ is a face of $V$ if and only if either $I$ is empty or $\mathbf{0}$ is in the relative interior of $\operatorname{conv} A_{V}(I)$.

Moreover, $V$ coincides with the vertex set of a convex polytope $P$ if and only if either

(i) $d=0$ (thus $P$ is a simplex) or

(ii) for every open halfspace $H^{+}$of $\mathbb{R}^{d}$ containing $\mathbf{0}$ in its closure, we have that $\operatorname{card}\left(\left\{i \mid A_{i} \in H^{+}\right\}\right) \geq 2$.

It follows that if $V$ is centered at the origin, then the double Gale transform of $V$ gives the same configuration in $\mathbb{R}^{m-d-1}$. However, this is not true in general (see Remark 5.5).

Based on the facts above, we have the following lemma (in which we use the same notation as in Proposition 5.1).

Lemma 5.2 Suppose that every vector of $V$ is a face, and every face of $V$ has at most $m-d-1$ vectors. Then $V$ coincides with the vertex set of a convex polytope $P$, whose boundary is simplicial. 
Proof Let $A_{V}=\left(A_{i}\right)_{i=1}^{m}$ be the Gale transform of $V$. It suffices to show either (i) or (ii) in Proposition 5.1 holds. Note that the case $d=0$ is trivial: this happens if and only if $V$ spans an $(m-1)$-simplex in $\mathbb{R}^{m-1}$.

Now suppose that $d>0$. Notice that by Proposition 5.1, the Siegel and weak hyperbolicity conditions hold for $A_{V}$, with the assumption above. Moreover, since every vector in $V$ is a face, we have $\mathbf{0} \in \operatorname{conv} A_{V}(J)$, for all $J$ with $\operatorname{card}(J)=m-1$.

Let $H^{+}$be an open halfspace of $\mathbb{R}^{d}$ with $\mathbf{0}$ on the boundary. From its admissibility, $A_{V}$ has rank $d$, with a neighborhood of $\mathbf{0} \in \mathbb{R}^{d}$ contained in $\operatorname{conv} A_{V}$ (see Lemma 3.1); hence there exists $A_{i} \in A_{V}$ such that $A_{i} \in H^{+}$. Observe that now $\mathbf{0} \in \operatorname{conv} A_{V}([m] \backslash\{i\})$ with $A_{V}([m] \backslash\{i\})$ again being admissible, by the same argument, there exists another $A_{j} \in A_{V}$ with $A_{j} \in H^{+}$, which means (ii) holds hence the statement follows.

Proposition 5.3 Let $K_{A}$ be the simplicial complex induced from an admissible $m-$ tuple $A=\left(A_{i}\right)_{i=1}^{m}$ centered at the origin, with vectors in $\mathbb{R}^{d}$. Let the tuple $V=\left(V_{i}\right)_{i=1}^{m}$ be the transpose of a basis of the system

$$
\left\{\begin{array}{l}
\sum_{i=1}^{m} A_{i} x_{i}=\mathbf{0}, \\
\sum_{i=1}^{m} x_{i}=0 .
\end{array}\right.
$$

Then $\left\{V_{i}\right\}_{\{i\} \in K_{A}}$ is the vertex set of a convex polytope $P_{A}$ of affine dimension $m-d-1$, with each $V_{j}$ in its interior, where $\{j\} \notin K_{A}$. Moreover, the boundary of $P_{A}$ is isomorphic to $K_{A}$ and we can assume that $P_{A}$ contains $\mathbf{0}$ in its interior.

Proof First from Lemma 3.1, the affine dimension of $V$ is $m-d-1$.

Since the centroid of $A$ is $\mathbf{0}$, now $A$ is the Gale transform of $V$, with the subtuple $\left(V_{i}\right)_{\{i\} \in K_{A}}$ satisfying the assumptions in Lemma 5.2; thus it coincides with the vertex set of a convex polytope $P_{A}$ whose boundary is simplicial. For those $\{j\} \notin K_{A}$, if $V_{j}$ lies outside, or on the boundary of $P_{A}$, it must be in a face of $V$ that is contained in a supporting hyperplane of $P_{A}$; by Proposition 5.1, this is impossible.

The last statement is also a consequence of Proposition 5.1, together with the observation that any translation of the form $V+v_{0}=\left(V_{i}+v_{0}\right)_{i=1}^{m}$ also satisfies (16).

Example 5.4 Let $A$ be the 5-tuple given by the matrix

$$
\left(\begin{array}{ccccc}
0 & 0 & 1 & 1 & -2 \\
1 & \frac{1}{2} & 0 & 0 & -\frac{3}{2}
\end{array}\right)
$$


which is admissible and centered at the origin. By solving (16) we can choose $V$ that is given by

$$
\left(\begin{array}{ccccc}
0 & 0 & -1 & 1 & 0 \\
6 & -9 & 2 & 0 & 1
\end{array}\right)
$$

Observe that the last point $(0,1)^{\mathrm{T}}$ is in the interior of the square spanned by the other four vertices.

Remark 5.5 Note that Proposition 5.3 is independent of the choice of $V$. If the centroid of $A$ is not at the origin, Proposition 5.3 may not hold. Consider the case that $A$ is given by the matrix (one can check its admissibility)

$$
\left(\begin{array}{cccc}
1 & 1 & 4 & -2 \\
4 & -2 & 1 & 1
\end{array}\right),
$$

then we choose $V=(-1,-1,1,1)$ by (16), but now points $V_{2}=(-1)$ and $V_{4}=(1)$ are not contained in the interior of $P_{A}=\operatorname{conv}\left(V_{1}, V_{3}\right)$. This is because the Gale transform of $V$ can be

$$
\left(\begin{array}{cccc}
0 & 0 & 1 & -1 \\
1 & -1 & 0 & 0
\end{array}\right),
$$

which is no longer admissible.

The following proposition is essentially due to Panov and Ustinovsky [18].

Proposition 5.6 Let $A=\left(A_{i}\right)_{i=1}^{m}$ be an admissible $m$-tuple centered at the origin. Then for each $z \in \mathcal{S}_{A}$ given, there is a unique pair $(r, T) \in \mathbb{R}_{>0} \times \mathbb{R}^{d}$ such that $\Phi_{A}(z, T, r)=r\left(z e^{\left\langle A_{i}, T\right\rangle}\right)_{i=1}^{m} \in\left(D^{2}, S^{1}\right)^{K_{A}}$ (see Section 4.1 for the definition).

Proof The proof which is presented here is adapted from Panov [17, Theorem 9.2, pages 37-40]. Observe that in the trivial case when $d=0$, ie $K_{A}$ is a simplex, we can simply take $r=\|z\|_{\infty}^{-1}$. In what follows suppose $d>0$.

Let $\mathbb{R}_{\geq 0}$ (resp. $\mathbb{R}_{\leq 0}$ ) be the set of nonnegative (resp. nonpositive) real numbers. Note that it suffices to prove the cases when $z \in\left(\mathbb{R}_{\geq 0}\right)^{m}$, since for each $z=\left(z_{i}\right)_{i=1}^{m} \in \mathbb{C}^{m}$, there is a rotation $e^{\sqrt{-1} \theta}=\left(e^{\sqrt{-1} \theta_{i}}\right)_{i=1}^{m} \in\left(\bar{S}^{1}\right)^{m}$ such that

$$
e^{\sqrt{-1} \theta} z=\left(e^{\sqrt{-1} \theta_{i}} z_{i}\right)_{i=1}^{m} \in\left(\mathbb{R}_{\geq 0}\right)^{m}
$$

and we have

$$
e^{\sqrt{-1} \theta} \Phi_{A}(z, T, r)=\Phi_{A}\left(e^{\sqrt{-1} \theta} z, T, r\right) .
$$

For the tuple $A=\left(A_{i}\right)_{i=1}^{m}$, let $V=\left(V_{i}\right)_{i=1}^{m}$ be the tuple defined in Proposition 5.3, which satisfies (16). Since $A$ is centered at the origin, the row vectors of $\widetilde{V}=\left(\tilde{V}_{i}\right)_{i=1}^{m}$ 
with $\tilde{V}_{i}=\left(V_{i}^{\mathrm{T}}, 1\right)^{\mathrm{T}}$ are a basis of the orthogonal complement of the space spanned by the row vectors of $A$.

Let $\alpha$ be the linear morphism

$$
\begin{gathered}
\alpha: \mathbb{R}^{m} \rightarrow \mathbb{R}^{m-d}, \\
\left(x_{i}\right)_{i=1}^{m} \mapsto \sum_{i=1}^{m} \tilde{V}_{i} x_{i} .
\end{gathered}
$$

For $x=\left(x_{i}\right)_{i=1}^{m} \in\left(\mathbb{R}_{\geq 0}\right)^{m}$, we shall abbreviate $\left(\ln \left(x_{i}\right)\right)_{i=1}^{m}$ as $\ln (x)$ in what follows.

First we consider the case $z \in \mathcal{S}_{A} \cap\left(\mathbb{R}_{>0}\right)^{m}$. Observe that there exists a pair $(r, T) \in$ $\mathbb{R}_{>0} \times \mathbb{R}^{d}$ such that $y=\left(y_{i}\right)_{i=1}^{m}=\Phi_{A}(z, T, r)$ when and only when $\ln (y)-w-\ln (z)=$ $\left(\left\langle A_{i}, T\right\rangle\right)_{i=1}^{m}$, where $w=\left(w_{i}\right)_{i=1}^{m}$ with $w_{i} \equiv \ln (r)$, and this happens if and only if the vector $\ln (y)-w-\ln (z)$ belongs to $\operatorname{Ker}(\alpha)$.

Let $\left(\mathbb{R}_{\leq 0}, 0\right)^{K_{A}}$ be the polyhedral product

$$
\left(\mathbb{R}_{\leq 0}, 0\right)^{K_{A}}=\bigcup_{\sigma \in K_{A}} D(\sigma), \quad D(\sigma)=\prod_{i=1}^{m} Y_{i}, Y_{i}= \begin{cases}\mathbb{R}_{\leq 0} & \text { if } i \in \sigma, \\ \{0\} & \text { otherwise }\end{cases}
$$

and it is clear that $y \in\left(D^{2}, S^{1}\right)^{K_{A}} \cap\left(\mathbb{R}_{>0}\right)^{m}$ if and only if $\ln (y) \in\left(\mathbb{R}_{\leq 0}, 0\right)^{K_{A}}$, hence now it suffices to find a unique pair $(u, c) \in\left(\left(\mathbb{R}_{\leq 0}, 0\right) K_{A}, \mathbb{R}\right)$, such that

$$
\sum_{i=1}^{m}\left(V_{i}^{\mathrm{T}}, 1\right)^{\mathrm{T}}\left(u_{i}+c\right)=\sum_{i=1}^{m}\left(V_{i}^{\mathrm{T}}, 1\right)^{\mathrm{T}} \ln \left(z_{i}\right)
$$

holds, where $u=\left(u_{i}\right)_{i=1}^{m}$. Let $\bar{P}_{A}$ be the convex polytope spanned by $\left\{-V_{i}\right\}_{\{i\} \in K_{A}}$. By Proposition 5.3, $\bar{P}_{A}$ contains a neighborhood of $\mathbf{0}$ in its interior, and the boundary of $\bar{P}_{A}$ is the union $-\bigcup_{\sigma \in K_{A}} \operatorname{conv} V(\sigma)$, which is simplicially isomorphic to $K_{A}$. Therefore every vector $v$ in $\mathbb{R}^{m-d-1}$ has a unique expression $\rho v_{0}$, where $\rho \in \mathbb{R}_{\geq 0}$ and $v_{0}$ lies in the relative interior of the corresponding face. Together with the observation $\sum_{i=1}^{m} V_{i}=\mathbf{0}$ (see (16)), we conclude that there exists a pair $(u, c) \in\left(\left(\mathbb{R}_{\leq 0}, 0\right)^{K_{A}}, \mathbb{R}\right)$ such that

$$
\sum_{i=1}^{m} V_{i} u_{i}=\sum_{\{i\} \in K_{A}} V_{i} u_{i}=\sum_{i=1}^{m} V_{i} \ln \left(z_{i}\right), \quad \sum_{i=1}^{m}\left(\ln \left(z_{i}\right)-u_{i}\right)=\sum_{i=1}^{m} c=m c,
$$

namely (18) holds, which is unique by the construction.

Next we consider general case when $z \in \mathcal{S}_{A} \cap \mathbb{R}_{\geq 0}^{m}$ with $\bar{I}_{z}=\left\{i \mid z_{i}=0\right\}$ not empty. First note that by definition, $\bar{I}_{z}$ is a simplex of $K_{A}$. Let $\pi_{z}: \mathbb{R}^{m-d-1} \rightarrow \mathbb{R}^{m-d-1-\operatorname{card}\left(\bar{I}_{z}\right)}$ 
be the orthogonal projection onto the linear subspace

$$
\bigcap_{i \in \bar{I}_{z}}\left\{v \in \mathbb{R}^{m-d-1} \mid\left\langle v, V_{i}\right\rangle=0\right\},
$$

and denote by $\operatorname{Link}\left(\bar{I}_{z}, K_{A}\right)$ the union

$$
\left\{\sigma \in K_{A} \mid\left(\sigma \cup \bar{I}_{z}\right) \in K_{A}, \sigma \cap \bar{I}_{z}=\varnothing\right\},
$$

which is a subcomplex of $\operatorname{Star}\left(\bar{I}_{z}, K_{A}\right)=\left\{\sigma \in K_{A} \mid \bar{I}_{z} \subset \sigma\right\}$. It is not difficult to see that in the image of $\pi_{z}, \pi_{z}\left(\operatorname{conv} V\left(\operatorname{Star}\left(\bar{I}_{z}, K_{A}\right)\right)\right.$ is a convex polytope bounded by $\pi_{z}\left(\operatorname{conv} V\left(\operatorname{Link}\left(\bar{I}_{z}, K_{A}\right)\right)\right.$ (for example, by induction on $\left.\operatorname{card}\left(\bar{I}_{z}\right)\right)$. Then by a similar argument as in the previous case, we deduce that there exists a unique $u=\left(u_{i}\right)_{i=1}^{m}$ in the polyhedral product $\left(\mathbb{R}_{\leq 0}, 0\right)^{\operatorname{Link}\left(\bar{I}_{z}, K_{A}\right)}$ (defined by replacing $K_{A}$ with $\operatorname{Link}\left(\bar{I}_{z}, K_{A}\right)$ in (17)), such that

$$
\pi_{z}\left(\sum_{i=1}^{m} V_{i} u_{i}\right)=\pi_{z}\left(\sum_{i=1}^{m} V_{i} \chi\left(z_{i}\right) \ln \left(z_{i}\right)\right), \quad \chi\left(z_{i}\right) \ln \left(z_{i}\right)= \begin{cases}\ln \left(z_{i}\right) & \text { if }\left|z_{i}\right|>0 \\ 0 & \text { otherwise }\end{cases}
$$

note that vectors of $\left\{V_{i}\right\}_{i \in \bar{I}_{z}}$ are linearly independent, hence we have a unique $x=$ $\left(x_{i}\right)_{i=1}^{m} \in \mathbb{R}^{m}$ with $I_{x} \subset \bar{I}_{z}$, such that

$$
\sum_{i=1}^{m} V_{i}\left(u_{i}+x_{i}\right)=\sum_{i=1}^{m} V_{i} \chi\left(z_{i}\right) \ln \left(z_{i}\right)
$$

holds. With $c$ obtained from

$$
\sum_{i=1}^{m}\left(\chi\left(z_{i}\right) \ln \left(z_{i}\right)-u_{i}-x_{i}\right)=m c
$$

we have

$$
\sum_{i=1}^{m}\left(V_{i}^{\mathrm{T}}, 1\right)^{\mathrm{T}}\left(u_{i}+x_{i}+c\right)=\sum_{i=1}^{m}\left(V_{i}^{\mathrm{T}}, 1\right)^{\mathrm{T}} \chi\left(z_{i}\right) \ln \left(z_{i}\right)
$$

At last, by solving $T \in \mathbb{R}^{d}$ from

$$
\left\langle A_{i}, T\right\rangle=\chi\left(z_{i}\right) \ln \left(z_{i}\right)-u_{i}-x_{i}-c
$$

for $i=1,2, \ldots, m$, and setting $r=e^{c}$, we have $\Phi_{A}(z, T, r) \in\left(D^{2}, S^{1}\right)^{K_{A}}$ as desired; the uniqueness follows from the arguments above and the observation that the rank of $A$ is $d$.

From Proposition 5.6, we can define a map $f_{\infty}: \mathcal{S}_{A} \rightarrow \mathcal{S}_{A}$, with $f_{\infty}(z)$ the point in the leaf $L_{z}$ such that $f_{\infty}(z) /\left\|f_{\infty}(z)\right\|_{\infty} \in\left(D^{2}, S^{1}\right)^{K_{A}}$. 
The proofs of Proposition 5.7 and Theorem 5.8 are similar to the ones for Proposition 3.4 and Theorem 3.5, respectively, which we shall omit here.

Proposition 5.7 With the assumption that $A$ is admissible and centered at the origin, the restriction $f_{2} /\left.\left\|f_{2}\right\|_{2}\right|_{\left(D^{2}, S^{1}\right)^{K_{A}}}:\left(D^{2}, S^{1}\right)^{K_{A}} \rightarrow X_{A}(2)$ is a homeomorphism, whose inverse is the restriction $f_{\infty} /\left.\left\|f_{\infty}\right\|_{\infty}\right|_{X_{A}(2)}: X_{A}(2) \rightarrow\left(D^{2}, S^{1}\right)^{K_{A}}$.

Theorem 5.8 The continuous function

$$
\begin{aligned}
\Phi_{A}(\infty):\left(D^{2}, S^{1}\right)^{K_{A}} \times \mathbb{R}^{d} \times \mathbb{R}_{>0} & \rightarrow \mathcal{S}_{A}, \\
(z, T, r) & \mapsto r\left(z_{i} e^{\left\langle A_{i}, T\right\rangle}\right)_{i=1}^{m},
\end{aligned}
$$

is a homeomorphism, provided that $A$ is admissible and centered at the origin.

Recall that for each $p \in[1, \infty)$, we have defined $T_{p}: \mathcal{S}_{A} \rightarrow \mathbb{R}^{d}$ such that $f_{p}(z)=$ $F\left(z, T_{p}(z)\right)$ has the minimal $L^{p}$-norm in each leaf $F_{z}$. By Theorem $3.5, T_{p}$ is the composition of $\Phi_{A}^{-1}(p)$ and the projection onto $\mathbb{R}^{d}$, and $f_{p}(z) /\left\|f_{p}(z)\right\|_{p}$ is the composition of $\Phi_{A}^{-1}(p)$ and the projection onto $X_{A}(p)$.

Corollary 5.9 Let $T_{\infty}: \mathcal{S}_{A} \rightarrow \mathbb{R}^{d}$ be the composition of $\Phi_{A}^{-1}(\infty)$ and the projection onto $\mathbb{R}^{d}$, with $A$ admissible and centered at the origin. Then we have

$$
\lim _{p \rightarrow \infty} T_{p}(z)=T_{\infty}(z)
$$

which means

$$
\lim _{p \rightarrow \infty} f_{p}(z) /\left\|f_{p}(z)\right\|_{p}=f_{\infty}(z) /\left\|f_{\infty}(z)\right\|_{\infty},
$$

with any $z \in \mathcal{S}_{A}$ given.

Proof By Lemma 4.1, Corollary 4.3 and Proposition 4.4, there exists a sequence $\left\{p_{k}\right\}_{k=1}^{\infty}$ such that $\left\{T_{p_{k}}(z)\right\}_{k=1}^{\infty}$ converges to a point $T_{0} \in \mathbb{R}^{d}$, and $\left\{f_{p_{k}}(z)\right\}_{k=1}^{\infty}$ converges to some $y_{0}$ such that $y_{0} /\left\|y_{0}\right\|_{\infty} \in\left(D^{2}, S^{1}\right)^{K_{A}}$. We claim that

$$
\lim _{p \rightarrow \infty} T_{p}(z)=T_{0}=T_{\infty}(z)
$$

with

$$
\lim _{p \rightarrow \infty} f_{p}(z) /\left\|f_{p}(z)\right\|_{p}=y_{0} /\left\|y_{0}\right\|_{\infty}=f_{\infty}(z) /\left\|f_{\infty}(z)\right\|_{\infty}
$$

Note that

$$
y_{0} /\left\|y_{0}\right\|_{\infty}=\lim _{k \rightarrow \infty} \Phi_{A}\left(z, T_{k}(z),\left\|f_{p_{k}}\right\|_{p_{k}}^{-1}\right)=\Phi_{A}\left(z, T_{0},\left\|y_{0}\right\|_{\infty}^{-1}\right) \in\left(D^{2}, S^{1}\right)^{K_{A}},
$$


which is uniquely determined by $z$ (see Proposition 5.6), therefore $y_{0} /\left\|y_{0}\right\|_{\infty}$ must be $f_{\infty}(z) /\left\|f_{\infty}(z)\right\|_{\infty}$ and $T_{0}$ must be $T_{\infty}(z)$. It is not difficult to see that the argument above is independent of the choice of the sequence $\left\{p_{k}\right\}_{k=1}^{\infty}$, hence the claim holds and the proof is completed.

\section{Some applications}

In this section we shall revisit several known results from another perspective. First notice that by Proposition 5.3, a simplicial complex $K_{A}$ induced from an admissible tuple that is centered at the origin can be realized as the boundary of a convex polytope dual to a simple one; the converse is also true: for a convex polytope with simplicial boundary, the Gale transform of its vertices will be a tuple with the property above.

Our first application is an alternative proof of a rigidity theorem on polytopal momentangle manifolds, due to Bosio and Meersseman:

Proposition 6.1 [3, Theorem 4.1] Let $K_{A}$ and $K_{A^{\prime}}$ be the simplicial complexes induced from two admissible $m$-tuples $A$ and $A^{\prime}$ that are centered at the origin, respectively. If there is a simplicial isomorphism $\phi: K_{A} \rightarrow K_{A}^{\prime}$, then there is a diffeomorphism between $X_{A}(2)$ and $X_{A^{\prime}}(2)$.

Proof Observe that under the assumption, $\phi$ can be extended as a bijection from $[\mathrm{m}]$ to itself (possibly not unique), and let $\widetilde{\phi}: \mathcal{S}_{A} \rightarrow \mathcal{S}_{A^{\prime}}$ be the diffeomorphism via permuting coordinates with respect to $\phi$. Clearly $\widetilde{\phi}$ gives a homeomorphism between associated moment-angle complexes $\left(D^{2}, S^{1}\right)^{K_{A}}$ and $\left(D^{2}, S^{1}\right)^{K_{A^{\prime}}}$. On the other hand, we have a smooth map $\left(f_{2}^{\prime} /\left\|f_{2}^{\prime}\right\|_{2}\right) \circ \widetilde{\phi}: X_{A}(2) \rightarrow X_{A^{\prime}}(2)$ given in the diagram

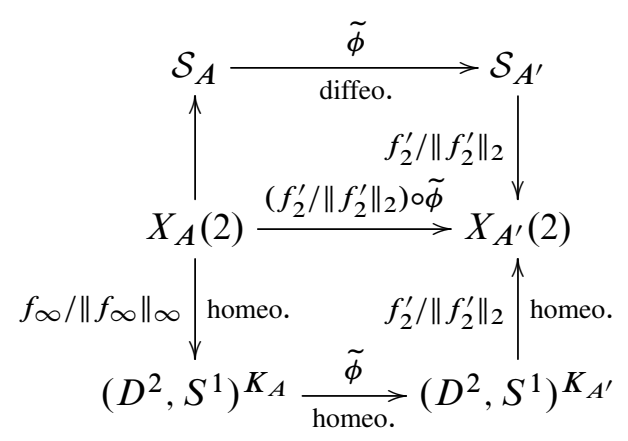

where $f_{2}^{\prime}: \mathcal{S}_{A^{\prime}} \rightarrow \mathcal{S}_{A^{\prime}}$ is the function of $L^{2}$-norm minima of Siegel leaves. By commutativity, it follows that $\left(f_{2}^{\prime} /\left\|f_{2}^{\prime}\right\|_{2}\right) \circ \widetilde{\phi}$ is a homeomorphism (see Theorem 1 and Proposition 5.7), whose inverse can be constructed by interchanging the roles of $A$ and $A^{\prime}$, which is also smooth. 
In what follows we shall discuss everything with $\mathbb{C}^{m}$ replaced by its subspace $\mathbb{R}^{m}$. In the foliation $\mathcal{F}$ given by the action (1), a leaf $L_{z}$ lies in $\mathcal{S}_{A} \cap \mathbb{R}^{m}$ if and only if $z \in \mathcal{S}_{A} \cap \mathbb{R}^{m}$. Therefore all properties hold true when restricted to the real case.

We will still use the same notation as in the previous sections, with the exception that the notation $\left(D^{1}, S^{0}\right)^{K_{A}}$ is used for the associated real moment-angle complex, ie the intersection of $\left(D^{2}, S^{1}\right)^{K_{A}}$ with $\mathbb{R}^{m}$ (see Section 4.1 for details).

Notice that the real version of Proposition 6.1 holds, namely the $\mathbb{Z}_{2}^{m}$-equivariant (where $\mathbb{Z}_{2}^{m}$ acts on $X_{A}(2)$ by changing the signs of coordinates) smooth structures on $X_{A}(2)$ are determined by combinatorial types of $K_{A}$. This can be deduced from a result of Wiemeler in [21, Corollary 5.2] (see also Davis [7, Corollary 1.3]).

Recall that a subspace $X$ of $\mathbb{R}^{m}$ is a polyhedron if for every point $x \in X$ there is a compact set $C_{X}$ such that $x * C_{X}=\left\{a x+b l \mid l \in C_{X}, a+b=1, a, b \geq 0\right\}$ is a neighborhood of $x$ in $X$. For instance, $\left(D^{1}, S^{0}\right)^{K_{A}}$ and $X_{A}(1)$ are polyhedra embedded in $\mathbb{R}^{m}$, hence they can be triangulated (see eg Rourke and Sanderson [19, Theorem 2.11]).

A polyhedron $X$ is a piecewise linear (abbreviated PL) $n$-manifold if given a certain triangulation, the link of each vertex is PL homeomorphic to the boundary of an $n$-simplex or to an $(n-1)$-simplex (ie these homeomorphisms become simplicial after suitable subdivisions on both sides). Note that this property is independent of the triangulation chosen for $X$ (see eg [19, pages 20-22]).

Definition 6.2 (Whitehead triangulation) Let $X$ be a polyhedron and $M$ a smooth manifold. A map $\eta: X \rightarrow M$ is a piecewise differentiable (abbreviated PD) homeomorphism if there exists a triangulation of $X$ such that the restriction of $\eta$ to each simplex is smooth with the Jacobian matrix nondegenerate. Such a PD homeomorphism $\eta$ is called a Whitehead triangulation of $M$, and also a smoothing of $X$.

Note that by Propositions 3.4 and 5.7, the smooth function $f_{2} /\left\|f_{2}\right\|_{2}: \mathcal{S}_{A} \rightarrow X_{A}(2)$ induces a homeomorphism when restricted to either $\left(D^{1}, S^{0}\right)^{K_{A}}$ or $X_{A}(1)$. Moreover, the following lemma holds:

Lemma 6.3 Let $A=\left(A_{i}\right)_{i=1}^{m}$ be an admissible tuple centered at the origin. If a space $Y \subset \mathbb{R}^{m}$ is either

(a) the intersection of the $L^{p}$-link $X_{A}(p)$ (defined by (6)) with the first orthant of $\mathbb{R}^{m}$ (ie points with nonnegative coordinates), for any $p \geq 1$, or

(b) a component of the polyhedral product $D(\sigma)=\left(D^{1}, S^{0}\right)^{\sigma}$ (see Section 4.1 for definition, with the pair replaced), for any $\sigma \in K_{A}$ with maximal dimension,

then $Y$ is a smooth manifold with corners, and the differential of $f_{2} /\left\|f_{2}\right\|_{2}$ at any point of $Y$ induces a linear injection between corresponding tangent spaces. 
Proof First we show that each $Y$ is indeed a smooth manifold with corners, in both cases. For (b) this is obvious since $Y$ is a cube of dimension $m-d-1$. As for (a), observe that for each $\sigma \in K_{A}$ with $\operatorname{card}(\sigma)=k$, the augmented subtuple $\tilde{A}([m] \backslash \sigma)$ has rank $d+1$, where $\tilde{A}=\left(\widetilde{A_{i}}\right)_{i=1}^{m}$ with $\tilde{A}_{i}=\left(A_{i}^{\mathrm{T}}, 1\right)^{\mathrm{T}}$ (see Lemma 3.1), therefore the row vectors of $\tilde{A}$, together with canonical basis vectors $e_{i} \in \mathbb{R}^{m}$ (the vector with only $i^{\text {th }}$ coordinate nonzero, which is one) for all $i \in \sigma$, form a matrix of rank $d+k+1$. This means that the intersection

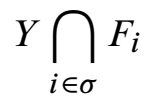

is transverse, where $F_{i}=\left\{\left(x_{i}\right)_{i=1}^{m} \in \mathbb{R}^{m} \mid x_{i}=0\right\}$.

Recall that $\Phi_{A}(2): X_{A}(2) \times \mathbb{R}^{d} \times \mathbb{R}_{>0} \rightarrow \mathcal{S}_{A}$ is a diffeomorphism such that $f_{2} /\left\|f_{2}\right\|_{2} \circ$ $\Phi_{A}(2)$ is the identity on $X_{A}(2)$ (see Theorem 1). Let

$$
\mathrm{d} \Phi_{A}(2)_{x}: \mathbb{R}^{m-d-1} \times \mathbb{R}^{d} \times \mathbb{R} \rightarrow \mathbb{R}^{m} \in T_{\Phi_{A}(2)(x)} \mathcal{S}_{A},
$$

be the differential of $\Phi_{A}(2)$ at the point $x$, and let $\zeta$ be the linear subspace $\{\boldsymbol{0}\} \times \mathbb{R}^{d} \times$ $\mathbb{R} \subset \mathbb{R}^{m}$ of dimension $d+1$. It suffices to show that for all $y=\left(y_{i}\right)_{i=1}^{m} \in Y$ with $x=\left(x_{i}\right)_{i=1}^{m}=f_{2}(y) /\left\|f_{2}(y)\right\|_{2}$, the intersection of the image of $\left.\mathrm{d} \Phi_{A}(2)_{x}\right|_{\zeta}$ with the tangent space $T_{y} Y$ is trivial.

For (a), note that from its definition (6), the tangent space $T_{y} Y$ is the orthogonal complement of the $(d+1)$-space spanned by the row vectors of the $((d+1) \times m)-$ matrix

$$
\tilde{A}_{y^{p-1}}=\left(\left(A_{i}^{\mathrm{T}}, 1\right)^{\mathrm{T}} y_{i}^{p-1}\right)_{i=1}^{m}
$$

and the image of $\left.\mathrm{d} \Phi_{A}(2)_{x}\right|_{\zeta}$ is spanned by the row vectors of $\tilde{A}_{y}=\left(\left(A_{i}^{\mathrm{T}}, 1\right)^{\mathrm{T}} y_{i}\right)_{i=1}^{m}$. From the previous argument, the subtuple $\tilde{A}_{y^{p-1}}\left(I_{y}\right)$ has rank $d+1\left(I_{y} \subset[m]\right.$ is the set of nonzero entries of $y$ ), hence any row vector of $\tilde{A}_{y}\left(I_{y}\right)$ cannot be orthogonal to the corresponding one in $\widetilde{A}_{y^{p-1}}\left(I_{y}\right)$, otherwise itself must be zero (since we can write each $y_{i}^{p}$ as a square).

As for (b), the tangent space at $y \in\left(D^{1}, S^{0}\right)^{\sigma}$ is spanned by $\left\{e_{i} \mid i \in \sigma\right\}$, where $\operatorname{card}(\sigma)=m-d-1$. But we have shown that the row vectors of $\tilde{A}_{y}\left(I_{y}\right)$ and the basis of $T_{y} Y$ has a full rank $m$, therefore the intersection of the image of $\left.\mathrm{d} \Phi_{A}(2)_{x}\right|_{\zeta}$ with $T_{y} Y$ must be trivial.

As a corollary, we find that with given triangulations, the restriction of $f_{2} /\left\|f_{2}\right\|_{2}$ to either $\left(D^{1}, S^{0}\right)^{K_{A}}$ or $X_{A}(1)$ will be a Whitehead triangulation of $X_{A}(2)$. By a theorem of Whitehead [20], if there is a PD homeomorphism from a polyhedron $X$ to a smooth manifold $M$, then $X$ is a PL manifold, and the PL structure on $X$ is 
uniquely determined by the smooth structure given on $M$. Consequently, it follows that $\left(D^{1}, S^{0}\right)^{K_{A}}$ and $X_{A}(1)$ are homeomorphic as PL manifolds.

At last, we make a conclusion to end this section.

Proposition 6.4 For each simplicial complex $K_{A}$ induced from an admissible $m-$ tuple $A$ centered at the origin, there is a PD homeomorphism from $\left(D^{1}, S^{0}\right)^{K_{A}}$ onto the smooth manifold $X_{A}(2)$, thus $\left(D^{1}, S^{0}\right)^{K_{A}}$ is a PL manifold of dimension $m-d-1$. If $\left(D^{2}, S^{1}\right)^{K_{A}}$ has an exotic PL structure, then either it is not smoothable, or $X_{A}(2)$ must have different smooth structures.

\section{References}

[1] A Bahri, M Bendersky, F R Cohen, S Gitler, The polyhedral product functor: A method of decomposition for moment-angle complexes, arrangements and related spaces, Adv. Math. 225 (2010) 1634-1668 MR2673742

[2] I V Baskakov, Triple Massey products in the cohomology of moment-angle complexes, Uspekhi Mat. Nauk 58 (2003) 199-200 MR2035723

[3] F Bosio, L Meersseman, Real quadrics in $\mathbb{C}^{n}$, complex manifolds and convex polytopes, Acta Math. 197 (2006) 53-127 MR2285318

[4] V M Buchstaber, T E Panov, Toric topology arXiv:1210.2368

[5] V M Buchstaber, T E Panov, Torus actions and their applications in topology and combinatorics, Univ. Lecture Series 24, Amer. Math. Soc. (2002) MR1897064

[6] C Camacho, N H Kuiper, J Palis, The topology of holomorphic flows with singularity, Inst. Hautes Études Sci. Publ. Math. (1978) 5-38 MR516913

[7] M W Davis, When are two Coxeter orbifolds diffeomorphic?, Michigan Math. J. 63 (2014) 401-421 MR3215556

[8] M W Davis, T Januszkiewicz, Convex polytopes, Coxeter orbifolds and torus actions, Duke Math. J. 62 (1991) 417-451 MR1104531

[9] G Denham, A I Suciu, Moment-angle complexes, monomial ideals and Massey products, Pure Appl. Math. Q. 3 (2007) 25-60 MR2330154

[10] S Gitler, S López de Medrano, Intersections of quadrics, moment-angle manifolds and connected sums, Geom. Topol. 17 (2013) 1497-1534 MR3073929

[11] M Goresky, R MacPherson, Stratified Morse theory, Ergeb. Math. Grenzgeb. 14, Springer, Berlin (1988) MR932724

[12] B Grünbaum, Convex polytopes, 2nd edition, Graduate Texts in Math. 221, Springer, New York (2003) MR1976856 
[13] S López de Medrano, Topology of the intersection of quadrics in $\mathbb{R}^{n}$, from: "Algebraic topology", (G Carlsson, R L Cohen, H R Miller, D C Ravenel, editors), Lecture Notes in Math. 1370, Springer, New York (1989) 280-292 MR1000384

[14] S López de Medrano, A Verjovsky, A new family of complex, compact, nonsymplectic manifolds, Bol. Soc. Brasil. Mat. 28 (1997) 253-269 MR1479504

[15] L Meersseman, A new geometric construction of compact complex manifolds in any dimension, Math. Ann. 317 (2000) 79-115 MR1760670

[16] L Meersseman, A Verjovsky, Holomorphic principal bundles over projective toric varieties, J. Reine Angew. Math. 572 (2004) 57-96 MR2076120

[17] T E Panov, Geometric structures on moment-angle manifolds, Uspekhi Mat. Nauk 68 (2013) 111-186 MR3113858 In Russian; translated in Russian Math. Surveys 68 (2013) 503-568

[18] T E Panov, Y Ustinovsky, Complex-analytic structures on moment-angle manifolds, Mosc. Math. J. 12 (2012) 149-172 MR2952429

[19] C P Rourke, B J Sanderson, Introduction to piecewise-linear topology, Ergeb. Math. Grenzgeb. 69, Springer, New York (1972) MR0350744

[20] J H C Whitehead, On $C^{1}$-complexes, Ann. of Math. 41 (1940) 809-824 MR0002545

[21] M Wiemeler, Exotic torus manifolds and equivariant smooth structures on quasitoric manifolds, Math. Z. 273 (2013) 1063-1084 MR3030690

Institute of Mathematics for Industry, Kyushu University

744 Motooka, Nishiku, Fukuoka 819-0395, Japan

l-cai@math.kyushu-u.ac.jp

Received: 11 April $2014 \quad$ Revised: 3 July 2014 